\title{
Percent Recovered Infinity Predicted
}

National Cancer Institute

\section{Source}

National Cancer Institute. Percent Recovered Infinity Predicted. NCI Thesaurus. Code C112035.

The percentage of the recovered administered dose extrapolated to infinity, calculated using the predicted value of the last non-zero concentration. 